\title{
C-5 Aryl Substituted Azaspirooxindolinones Derivatives: Synthesis and Biological Evaluation as Potential Inhibitors of Tec Family Kinases
}

\author{
Gopal Mudasani ${ }^{\mathrm{a}, \mathrm{b}}$, Kalyani Paidikondala ${ }^{\mathrm{a}}$, Soňa Gurskác, Shambabu Joseph Maddirala ${ }^{\mathrm{b}}$, Petr \\ Džubák $^{\mathrm{c}}$, Viswanath Das ${ }^{\mathrm{c}, *}$, Rambabu Gundla $^{\mathrm{a}, *}$ \\ aDepartment of Chemistry, School of Science, GITAM (Deemed to be University, Hyderabad 502102, \\ Telangana, India \\ ${ }^{b}$ Medicinal Chemistry Laboratory Division, Aragen Lifesciences Pvt. Ltd, Survey No: 125(Part) \& 126, IDA \\ Mallapur, Hyderabad - 500 076, India. \\ 'Institute of Molecular and Translational Medicine, Olomouc, Hněvotínská 1333/5, 77900 Olomouc, Czech \\ Republic
}

\begin{abstract}
The interleukin-2-inducible kinase (ITK) and Bruton tyrosine kinase (BTK) are two crucial Tec family kinase members with important roles in the development of hematopoietic malignancies, autoimmune disorders and other diseases in human. Thus, ITK and BTK are key targets for drug development. Spirooxindoles are important scaffolds for the synthesis of small molecules with broad and potent biological activities. In this study, we performed a structure-activity relationship study of a new series of 5'-(benzo[d][1,3]dioxol-5yl)spiro[piperidine-4,3'-pyrrolo[2,3-b]pyridin]2'(1'H)-one linked with $\mathrm{N}$-acyl and $\mathrm{C}-5$ arylsubstituted scaffolds in a panel of ITK and BTK
\end{abstract}

\section{Introduction}

Spirooxindoles, with heterocyclic motifs attached at the C-3 position of the oxindole core, are promising candidates in drug discovery and development. Reports suggest that spirooxidoles fused with different cyclic rings exhibit significant anticancer activity against selected cell lines. Likewise, spirooxindolecyclopropane analogues [1], spirooxindolepyrrolidine derivatives [2], spirooxindolecyclohexane substituents fused with a pyrrole ring $[3,4]$ and morpholine fused with 1,2,3- cancer cell lines. Four compounds 11, 12, 14 and 15 showed high antiproliferative activity against ITK and BTK cell lines. Compounds 11 and 12 with a C-5 benzodioxole group and gem-dialkyl group attached to carbonyl on piperidine were highly effective in ITK-high Jurkat and CEM cell lines, and compound 14, a biotin analogue, was identified as a good inhibitor of BTK-high RAMOS cells. Compound 15 with cyclopropyl group attached to carbonyl on piperidine also showed good activity in ITK and BTK cell lines.

Keywords: Antiproliferative activity; Bruton tyrosine kinase; CCRF-CEM cells; Interleukin-2inducible kinase; Jurkat cells; K562 cells, RAMOS cells, Azaspirooxidole, Biotin, Suzuki-coupling reaction.

triazoles [5] exhibit a wide range of anticancer activity. The symmetrical trispirooxindole (JW67) is also effective against human SW480 colon adenocarcinoma cells through its effects on $\beta$-catenin levels [6]. Balan Balan et al. in 2020 reported that the substitution on piperidine nitrogen of spirooxindole moiety $\mathbf{V}$ results in derivates with nanomolar anticancer activity against HPK1 cell lines [7]. Further, C-5 substitution of spirooxindole derivatives of VI exhibited good anticancer activity [8]. The synthesised spirooxindole derivative such as

\footnotetext{
* Correspondence to: Viswanath Das (viswanath.das@upol.cz) and Rambabu Gundla (rgundla@gitam.edu)
} 
MI-888 has been in preclinical research to treat human cancers [9].

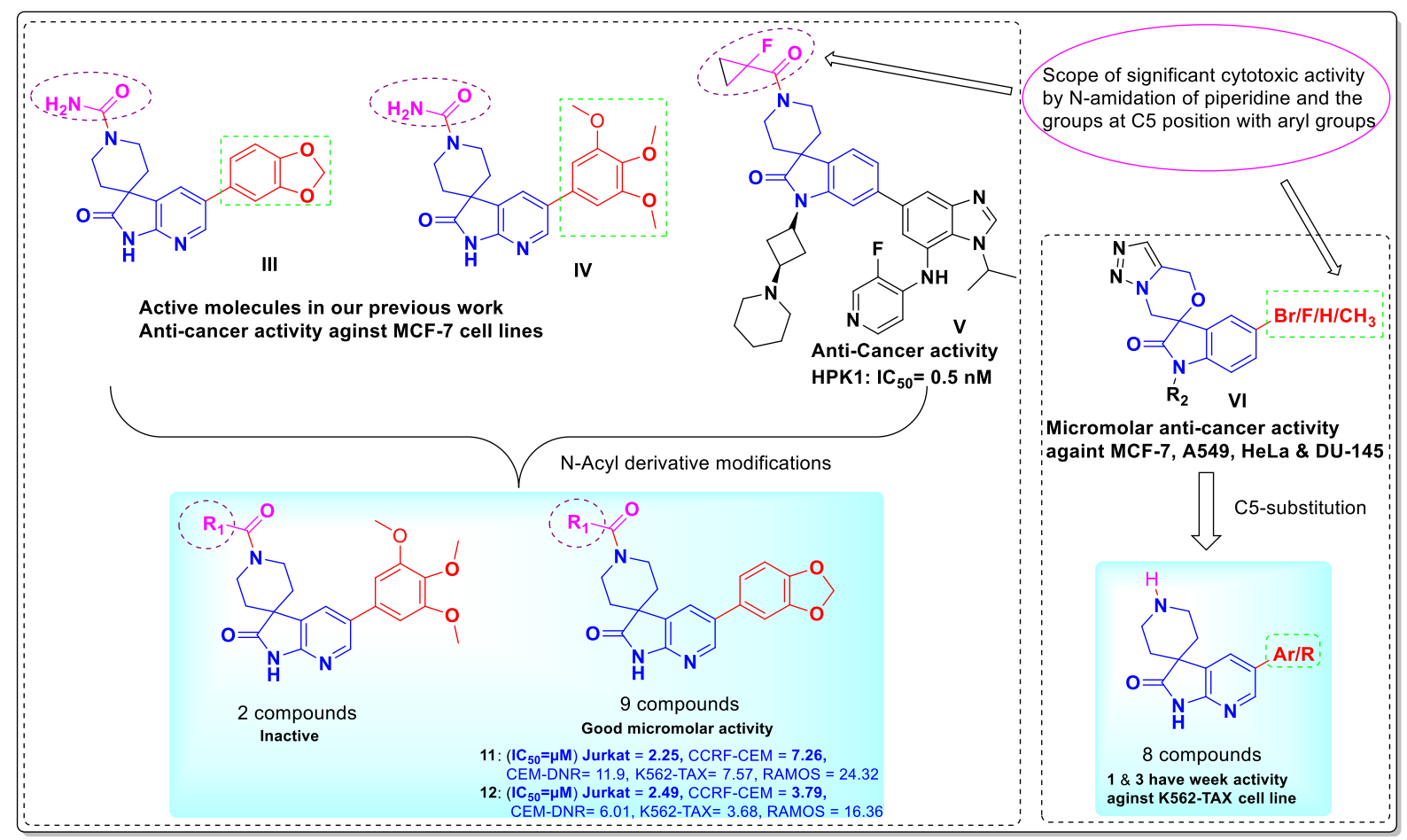

Fig. 1. Design of new series of azaspirooxindole analogues

The interleukin-2-inducible kinase (ITK) is a non-receptor protein tyrosine kinase that belongs to the Tec kinase family [10]. Although predominantly expressed in T-cell lineages, natural killer cells and mast cells are also known to express ITK [10]. By modulating calcium signalling, ITK controls the activity and translocation of transcription factors like NFAT and NFкB [11]. ITK drug discovery and development is becoming an attractive research area due to ITK's role in T-cell malignancies, autoimmune disorders and other human diseases [11-15]. Some prominent ITKspecific molecules developed since the early 2000 s to target the active site include aminothiazoles, benzimidazoles, indoles, thienopyrazoles, imidazopyridines, and pryridones [16-22]. Other newly designed ITK inhibitors include aryl ketones by Mankind Pharma [23] and aminobenzothiazole by
GlaxoSmithKline plc [24]. Furthermore, several diverse molecules such as 2-amino-5(thioaryl)thiazoles, 2-amino-5[(thiomethyl)aryl] thiazoles, (4 or 5aryl)pyrazolyl-indoles, and benzimidazole derivatives have been reported as potent ITK inhibitors [25,26]. PRN694, a benzimidazol, is an ATP site-binding covalent inhibitor of ITK and receptor-like kinase but not Bruton tyrosine kinase (BTK) [27]. PRN694 is a potential drug for preventing colitis disease and psoriasis therapy [28].

Although there is tremendous interest in ITK as a drug target, only a few novel ITK inhibitors exist in preclinical and clinical trials [10]. Currently, ibrutinib is the only approved treatment for chronic lymphocytic leukaemia and mantle B-cell lymphoma in the European Union since 2012 [29]. Despite their biological significance, spirooxindoles and their 
derivatives have not been explored extensively as anti-ITK compounds. Encouraged by the moderate activity of compounds III and IV against MCF-7 cell lines in our previous work [30] and structural optimisation of ibrutinib with benzodioxole group in another study [31], we herein report a structure-activity relationship study of two sets of azaspiroindolinones derivatives. First, a set of eight compounds (1-8) were synthesised to identify the activity of different hydrophilic groups substituted on aryl moiety on the C5 position of azaspirooxindolinone ring (Fig. 1). Next, the second series of eleven compounds (9-19) were prepared based on compound III and IV to evaluate the importance of carboxylic acid substituents on piperidine nitrogen by the conventional acid-amine coupling method. Altogether, we have synthesised nineteen new azaspiroindolinones and evaluated in vitro anticancer activity against a panel of ITK and BTK cell lines.

\section{Results \& Discussions}

\subsection{Chemistry}

The key compounds I, III and IV were prepared as previously reported [30]. The 7azaspirooxindole scaffold $\mathbf{I}$ can be easily substituted with functional groups at the C5 position by standard synthetic chemistry, allowing rapid exploration of the structureactivity relationship (SAR). Scheme 1 illustrates a two-step general synthetic route for the preparation of $\mathbf{1}, \mathbf{2}, 3,4,6$ and 8 . To synthesise these compounds, we performed Suzuki-coupling between compound I and commercially available aryl boronic acid in the presence of trans $-\mathrm{PdCl}_{2}\left(\mathrm{PPh}_{3}\right)_{2}$ catalyst and $\mathrm{NaHCO}_{3}$ in acetonitrile-water at $90^{\circ} \mathrm{C}$ to give the intermediate $\mathrm{C}$-arylation compounds. These intermediates on further global deprotection of Boc and SEM groups with trifluoroacetic acid resulted in corresponding compounds. Further, de-methylation of compound $\mathbf{6}$ was attempted with $\mathrm{BBr}_{3}$ in dichloromethane, giving compound 7 in moderate yield.

The Heck-coupling of arylbromo compound I and tert-butyl acrylate using trans$\mathrm{PdCl}_{2}\left(\mathrm{PPh}_{3}\right)_{2}$ as the catalyst and triethylamine in toluene at $100^{\circ} \mathrm{C}$ under microwave irradiation yielded the intermediate II [32]. This intermediate II on further treatment with trifluoroacetic acid resulted in compound $\mathbf{5}$ (Scheme 2) as a white solid.

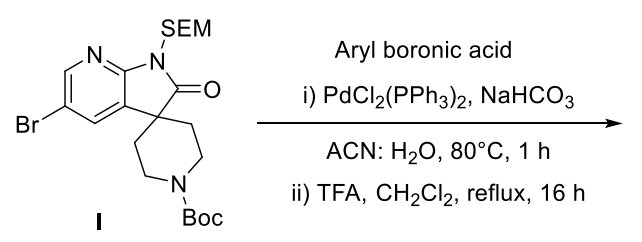

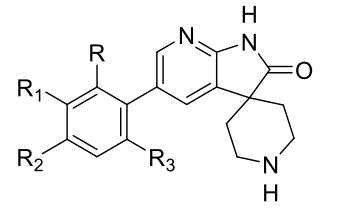

1; R, R, $\mathrm{R}_{3}=\mathrm{H} ; \mathrm{R}_{2}=\mathrm{OCH}_{3}$

2; $R, R_{3}=H, R_{1} ; R_{2}=F$

3; $\mathrm{R}, \mathrm{R}_{2}, \mathrm{R}_{3}=\mathrm{H} ; \mathrm{R}_{1}=\mathrm{CN}$

4; $\mathrm{R}, \mathrm{R}_{1}, \mathrm{R}_{3}=\mathrm{H} ; \mathrm{R}_{2}=\mathrm{OH}$

6; $\mathrm{R}, \mathrm{R}_{3}=\mathrm{H} ; \mathrm{R}_{1}, \mathrm{R}_{2}=\mathrm{OCH}_{3}$

8; $R_{1}, R_{2}=H ; R, R_{3}=F$

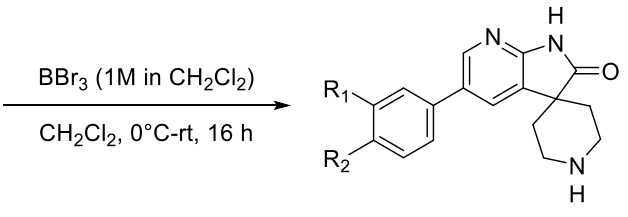

7; $\mathrm{R}_{1}, \mathrm{R}_{2}=\mathrm{OH}$

Scheme 1. Synthetic scheme for compounds 1, 2, 3, 4, 6, 7 and 8. 
To synthesise compounds 9-12, 14, 15, 16, 18 and 19, the key building block III was prepared with the same protocol reported in our previous work [30] and coupled with various carboxylic acids using HATU reagent, N, Ndiisopropylethylamine in DMF at ambient temperature give the target compounds in good yield (Scheme 3).
Substitution of compound IV with commercially available NHS-biotin using triethylamine base in DMF at room temperature gives compound $\mathbf{1 3}$ as a white solid. Similarly, III produces compound $\mathbf{1 4}$ as a white solid. The general acid-amine coupling of compound IV with 1-(methoxycarbonyl)cyclopropane-1carboxylic acid with HATU/DIPEA obtained 17 in good yield. (Scheme 4).<smiles>C#[M]N1C(=O)C2(CCN(C(=O)OC(C)(C)C)CC2)c2cc(Br)cnc21</smiles>

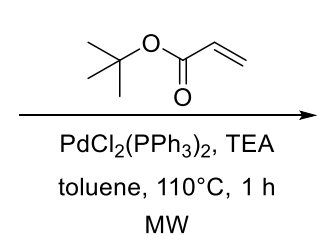<smiles>CC(C)(C)OC(=O)/C=C/c1cnc2c(c1)C1(CCN(C(=O)OC(C)(C)C)CC1)C(=O)N2[Si]#N</smiles>

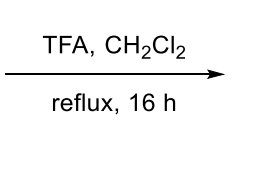<smiles>O=C(O)/C=C/c1cnc2c(c1)C1(CCNCC1)C(=O)N2</smiles>

Scheme 2. Synthetic scheme of compound 5.

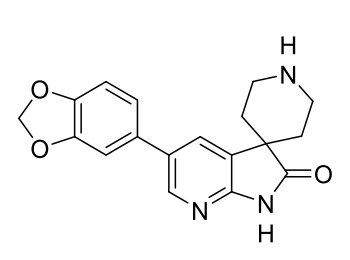

III

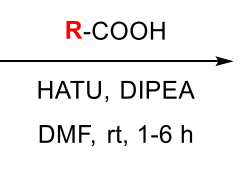

DMF, rt, 1-6 h

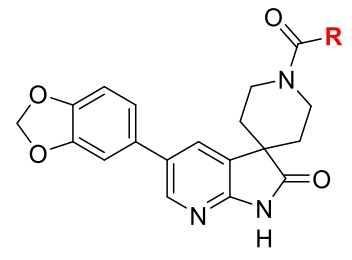

$9-12,14,15,16,18 \& 19$

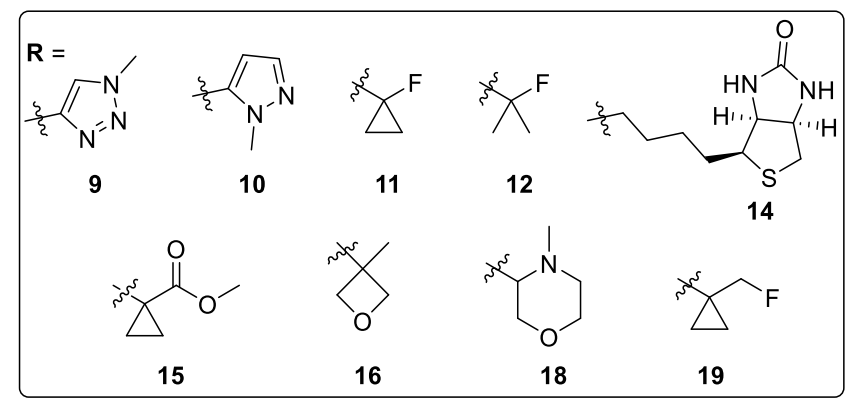

Scheme 3. Synthetic scheme for compounds 9-12, 14, 15, 16, 18 and 19. 

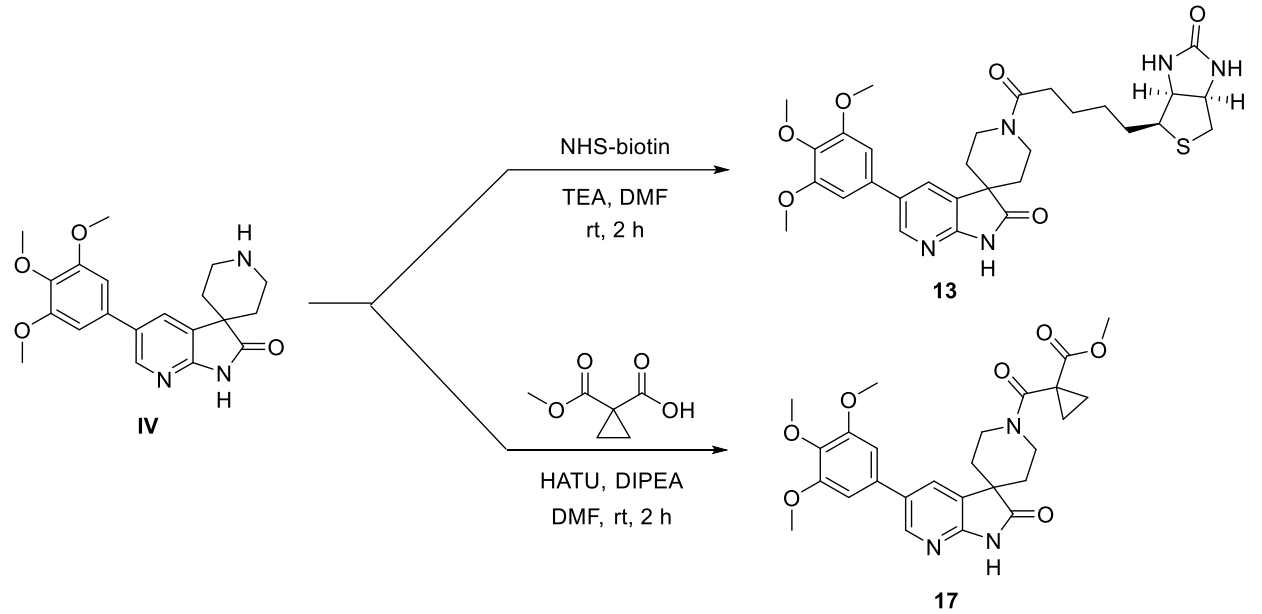

Scheme 4. Synthetic scheme for compounds 13 and 17.

\subsection{Biology}

Nineteen derivatives were tested in a panel of cell lines consisting of ITK-high T-cell leukaemia lines [33,34], BTK-high B-cell leukaemia lines [33,35], ITK/BTK-null malignant lines, and two nonmalignant fibroblast lines. The compounds that did not result in 50\% inhibition of cell proliferation when tested at a single dose of $50 \mu \mathrm{M}$ were not processed further for dose-response analysis (Table 1, 2). For the cytotoxicity profiling, compounds are generally regarded as inactive if the $\mathrm{IC}_{50}$ is above $50 \mu \mathrm{M}$, weakly active above $30 \mu \mathrm{M}$, moderately active between 10 to $20 \mu \mathrm{M}$, and highly active below $10 \mu \mathrm{M}$. Based on this standard convention, the effect of six structurally similar compounds (active - 11, 12, 14, 15; and inactive -13 and 17) were interesting to observe in ITK and BTK-high cell lines compared to ITK/BTK-null cells (Table 2 ). The active compounds were inactive in nonmalignant cells or showed weak activity in U2OS and HCT116 cells.

The SAR profiling indicates that group benzodioxole at the $\mathrm{C} 5$ position in compounds 11, 12 and 15, and 1-fluorocyclopropane in 11, gem-dimethyl-1-fluoro in $\mathbf{1 2}$ and methyl-1cyclopropane-1-carboxylate in $\mathbf{1 5}$ attached to carbonyl $(\mathrm{C}=\mathrm{O})$ are essential for biological activity (Table 2). The $\mathrm{N}$-acyl analogue in 1methyl-1H-1,2,3-triazole compound 9 did not enhance the antiproliferative activity. Compound 10 with 1-methyl-1H-1,2,3-triazole is inactive across all the cell lines. Compound 15 that possessed cyclopropyl attached with electron-withdrawing methyl ester group was active against most tested anticancer cell lines. This compound exhibited good anticancer activity against ITK high cell lines $\left(\mathrm{IC}_{50}=14.8\right.$ $28.8 \mu \mathrm{M}$ ), moderate activity in BTK high cell lines $\left(\mathrm{IC}_{50}=12.8-34.8 \mu \mathrm{M}\right)$ and weak activity in ITK/BTK null cell lines $\left(\mathrm{IC}_{50}=37.7-49.2 \mu \mathrm{M}\right)$. Substitution of C5 position in 17 with $3,4,5-$ trimethoxy phenyl group affected compound activity in all anticancer cell lines. Similarly, compound 13, a 3,4,5-trimethoxy phenylsubstituted at $\mathrm{C} 5$ analogue of active compound $14\left(\right.$ RAMOS IC IC $_{50}=9.0 \mu \mathrm{M}$; Jurkat $\mathrm{IC}_{50}=26.0$ $\mu \mathrm{M}$ ), did not possess any antiproliferative activity in tested cell lines. Increasing the ring size by introducing oxetane ring with the hydrophobic tert-methyl group at $\mathrm{N}$-acyl carbonyl in $\mathbf{1 6}$ did not improve the activity against ITK cell lines (CCRF-CEM IC I0 $_{50}=33.4$ $\mu \mathrm{M}$; CEM-DNR $\left.\mathrm{IC}_{50}=49.7 \mu \mathrm{M}\right)$. Compound 18 with a six-membered $\mathrm{N}$-methylmorpholine was inactive in all cell lines. This result 
indicates that cyclopropyl or a similar gemdimethyl group on $\mathrm{N}$-acyl carbonyl of spiropiperidine ring is essential for the activity of this class of compounds. As expected, compound 19, resulting from fluoromethylcyclopropane, was also moderately active in ITK high cell lines $\left(\mathrm{CCRF} \mathrm{CEM} \mathrm{IC}_{50}=25.5 \mu \mathrm{M} ; \mathrm{CEM}-\mathrm{DNR} \mathrm{IC}_{50}\right.$ $=28.9 \mu \mathrm{M}$ ).

Surprisingly, structural activity relationship profiling for C5 aryl substituent motifs with electron-rich atoms/groups such as 3,4difluorophenyl (2), 2,6-difluorophenyl (8), 4hyrdoxy phenyl analogue (4), 3,4dimethoxyphenyl (6) and 3,4-dihydroxyphenyl substituent $\mathbf{7}$ without any group on the piperidine nitrogen were inactive against all cell lines. Compound 1 with 3-methoxyphenyl and compound $\mathbf{3}$ with 3-cyanophenyl group were poorly active against K562-tax cells. The C5 alkyl substitution compound $\mathbf{5}$ with a double bond is inactive in all cell lines.

Table 1. IC 50 values of compounds 1-4 and 6-8 in cancer and non-cancer cell lines. Mean \pm SD of at least 3 independent experiments.

\begin{tabular}{|c|c|c|c|c|c|c|c|c|c|c|c|c|c|c|}
\hline \multirow[b]{2}{*}{ ipd } & \multirow[b]{2}{*}{ :, $\mathbf{R}_{3}$} & \multirow[b]{2}{*}{$\mathbf{R}_{\mathbf{1}}$} & \multirow[b]{2}{*}{$\mathrm{R}_{2}$} & \multicolumn{11}{|c|}{$\mathrm{IC}_{50}(\mu \mathrm{M})$} \\
\hline & & & & $\begin{array}{c}\text { BJ, } \\
\text { MRC-5 }\end{array}$ & A549 & НСТ116 & $\begin{array}{c}\text { :T116 p53- } \\
\text { /- }\end{array}$ & U2OS & RAMOS & K562 & 62-TAX & M-DNR & IRF-CEM & Jurkat \\
\hline & & & & & \multicolumn{4}{|c|}{ ITK/BTK null } & \multicolumn{3}{|c|}{ BTK high } & \multicolumn{3}{|c|}{ ITK high } \\
\hline 1 & $H$ & OMe & $H$ & $>50$ & $>50$ & $>50$ & $>50$ & $>50$ & $>50$ & $>50$ & 44.5 & $>50$ & $>50$ & $>50$ \\
\hline 2 & $H$ & $F$ & $F$ & $>50$ & $>50$ & $>50$ & $>50$ & $>50$ & $>50$ & $>50$ & $>50$ & $>50$ & $>50$ & $>50$ \\
\hline 3 & $H$ & $C N$ & $H$ & $>50$ & $>50$ & $>50$ & $>50$ & $>50$ & $>50$ & $>50$ & 46.2 & $>50$ & $>50$ & $>50$ \\
\hline 4 & $H$ & $H$ & $\mathrm{OH}$ & $>50$ & $>50$ & $>50$ & $>50$ & $>50$ & $>50$ & $>50$ & $>50$ & $>50$ & $>50$ & $>50$ \\
\hline 6 & $H$ & OMe & OMe & $>50$ & $>50$ & $>50$ & $>50$ & $>50$ & $>50$ & $>50$ & $>50$ & $>50$ & $>50$ & $>50$ \\
\hline 7 & $H$ & $\mathrm{OH}$ & $\mathrm{OH}$ & $>50$ & $>50$ & $>50$ & $>50$ & $>50$ & $>50$ & $>50$ & $>50$ & $>50$ & $>50$ & $>50$ \\
\hline 8 & $\mathrm{~F}$ & $\mathrm{H}$ & $\mathrm{H}$ & $>50$ & $>50$ & $>50$ & $>50$ & $>50$ & $>50$ & $>50$ & $>50$ & $>50$ & $>50$ & $>50$ \\
\hline
\end{tabular}

\section{Conclusion}

We have reported two sets of azaspiroindolinone derivatives and reported high activity of 4 structurally similar compounds against ITK and BTK positive cell lines. The active compounds $\mathbf{1 1}$ and $\mathbf{1 2}$ displayed a higher activity against ITK positive lines and moderate activity in BTK lines, whereas 14 was highly active in BTK than ITK cell lines. Members of the Tec kinase family share similarities in structure, consisting of $\mathrm{PH}$ domain, $\mathrm{SH} 3$ domain, $\mathrm{SH} 2$ domain and kinase domain [10]. The selectivity of active compounds against ITK and other members of the Tec kinase family is currently being planned for the identified active compounds. Further, coupling with aryl or alkyl groups on spiropiperidine amide (-NH) can generate derivatives with higher specificity against ITK and/or BTK. Nonetheless, given the role of immune cell-mediated human disorders [10], our findings provide new scaffolds for 
stimulating the development of therapeutics directed against ITK and BTK. 
Table 2. $\mathrm{IC}_{50}$ values of compounds 9-19 in cancer and non-cancer cell lines. Mean \pm SD of at least 3 independent experiments.

\begin{tabular}{|c|c|c|c|c|c|c|c|c|c|c|c|c|}
\hline \multirow{2}{*}{ Cmpd. } & \multirow{2}{*}{$\begin{array}{l}\text { Substituent } \\
\text { (R) }\end{array}$} & \multicolumn{11}{|c|}{$\mathrm{IC}_{50}(\mu \mathrm{M})$} \\
\hline & & \multirow[t]{2}{*}{$\begin{array}{c}\text { BJ, } \\
\text { MRC-5 }\end{array}$} & 1549 & HCT116 & $\begin{array}{c}\text { HCT116 } \\
\text { p53-/- }\end{array}$ & U2OS & RAMOS & K562 & $\begin{array}{l}\text { K562- } \\
\text { TAX }\end{array}$ & $\begin{array}{l}\text { CEM- } \\
\text { DNR }\end{array}$ & $\begin{array}{l}\text { CCRF- } \\
\text { CEM }\end{array}$ & Jurkat \\
\hline & & & \multicolumn{4}{|c|}{ ITK/BTK null } & \multicolumn{3}{|c|}{ BTK high } & \multicolumn{3}{|c|}{ ITK high } \\
\hline 9 & & $>50$ & 50 & $>50$ & $>50$ & $>50$ & $>50$ & $>50$ & 22.4 & 43.2 & 45.9 & $>50$ \\
\hline 10 & & $>50$ & 50 & $>50$ & $>50$ & $>50$ & $>50$ & $>50$ & $>50$ & $>50$ & $>50$ & $>50$ \\
\hline 11 & & $>50$ & $>50$ & $>50$ & $>50$ & 36.2 & 24.3 & $>50$ & 7.6 & 11.9 & 7.3 & 2.3 \\
\hline 12 & & $>50$ & $>50$ & $>50$ & $>50$ & 30.3 & 16.4 & $>50$ & 3.9 & 6.0 & 3.8 & 2.5 \\
\hline 13 & & $>50$ & $>50$ & $>50$ & $>50$ & $>50$ & $>50$ & $>50$ & 50 & $>50$ & $>50$ & $>50$ \\
\hline 14 & & $>50$ & $>50$ & $>50$ & $>50$ & $>50$ & 9.0 & $>50$ & $>50$ & $>50$ & 29.8 & 26.0 \\
\hline 15 & & $>50$ & $>50$ & 37.7 & 41.6 & 49.2 & 24.0 & 34.8 & 12.5 & 20.5 & 15.2 & 14.8 \\
\hline 16 & & $>50$ & 750 & $>50$ & $>50$ & $>50$ & $>50$ & $>50$ & 50 & 49.7 & 33.2 & $>50$ \\
\hline 17 & & $>50$ & $>50$ & $>50$ & $>50$ & $>50$ & $>50$ & $>50$ & 50 & $>50$ & $>50$ & $>50$ \\
\hline 18 & & $>50$ & $>50$ & $>50$ & $>50$ & $>50$ & $>50$ & $>50$ & $>50$ & $>50$ & $>50$ & $>50$ \\
\hline 19 & 2 & $>50$ & 50 & $>50$ & $>50$ & $>50$ & $>50$ & $>50$ & 30.7 & 28.9 & 25.5 & $>50$ \\
\hline
\end{tabular}

\subsection{Experimental section}

\subsection{Chemistry}

\subsubsection{Materials and Methods}

All chemicals (reagent grade) used were purchased from Combi-Blocks (USA), Johnson Matthey Co., Ltd., (USA) and Enamine Ltd. (Ukraine). All the solvents used for the reaction are LR grade. Analytical thin-layer chromatography (TLC.) was performed on 
precoated silica gel $60 \mathrm{~F}_{254}$ plates, and visualisation on TLC was achieved by UV light. Flash column chromatography was undertaken on silica gel (100-200 mesh). ${ }^{1} \mathrm{H}$ NMR was recorded on 400 or $500 \mathrm{MHz}$, and chemical shifts were quoted in parts per million (ppm) referenced to $0.0 \mathrm{ppm}$ for tetramethylsilane. The following abbreviations were used to describe peak splitting patterns when appropriate: $\mathrm{br}=$ broad, $\mathrm{s}=$ singlet, $\mathrm{d}=$ doublet, $\mathrm{t}=$ triplet, $\mathrm{q}=$ quartet, $\mathrm{m}=$ multiplet, $\mathrm{dd}=$ doublet of doublet. Coupling constants, $J$, were reported in the hertz unit (Hz). API LC-mass spectra were obtained on Agilent and Waters instruments. All the final compounds were purified on GRACE flash chromatography by using $\mathrm{C} 18$ reverse-phase columns. The mobile phase was a mixture of water $(0.1 \%$ formic acid) and acetonitrile. Melting points were recorded on the Buchi M-560 instrument.

\subsubsection{General method A}

A solution mixture of arylbromo compound I (1.0 eq, $0.15 \mathrm{mmol}$ ), aryl boronic acid (1.5 eq.), $\mathrm{PdCl}_{2}\left(\mathrm{PPh}_{3}\right)_{2}$ (0.05 eq), X-Phos (0.1 eq.) and $\mathrm{NaHCO}_{3}$ (2.0 eq) in acetonitrile: water (8:2) $(4.0 \mathrm{~mL})$ were de-gassed with argon for about 5 min. The resulting reaction mixture was maintained under stirring at $90{ }^{\circ} \mathrm{C}$ for $1-3 \mathrm{~h}$. The reaction mixture was allowed to room temperature, diluted with ethyl acetate (25-30 $\mathrm{mL}$ ), added $0.2 \mathrm{~g}$ of activated charcoal and filtered through celite. The filtrate was partitioned between water and ethyl acetate. The combined ethyl acetate layer was washed with brine, dried over anhydrous $\mathrm{Na}_{2} \mathrm{SO}_{4}$ and concentrated. The crude was purified by column chromatography over silica gel (100200 mesh) using a solvent gradient mixture of $20-60 \%$ ethyl acetate in pet-ether as an eluent to afford the desired $\mathrm{C}$-aryl compound. A mixture of the intermediate compound $(0.1 \mathrm{mmol})$ and TFA $(2.0 \mathrm{~mL})$ in dichloromethane $(2.0 \mathrm{~mL})$ was heated to $60^{\circ} \mathrm{C}$ for $16 \mathrm{~h}$ and distilled off the volatiles under reduced pressure. The crude residue was purified by GRACE flash chromatography using a C18 column with $0.1 \%$ formic acid in water and acetonitrile as an eluent to obtain the final compound.

\subsubsection{General method B}

To a solution of acid (1.0 eq.), HATU (1.5 eq.) and DIPEA (3.0 eq.) in DMF (0.2 M) was added amine (1.2 eq.) at room temperature. The reaction mixture was stirred at room temperature for $2 \mathrm{~h}$. The reaction mixture was partitioned between water $(30 \mathrm{~mL})$ and ethyl acetate $(30 \mathrm{~mL} \mathrm{X} \mathrm{3).} \mathrm{The} \mathrm{combined} \mathrm{organic}$ layer was washed with water $(50 \mathrm{~mL})$, brine $(50$ $\mathrm{mL}$ ), dried over anhydrous $\mathrm{Na}_{2} \mathrm{SO}_{4}$ and concentrated. The crude was purified by GRACE flash chromatography using a C18 column with $0.1 \%$ formic acid in water and acetonitrile as an eluent to give the final compound.

4.2. Synthesis of compounds 1, 2, 3, 4, 6 and 8 (Following general method $\mathbf{A}$ )

4.2.1 5'-(4-Methoxyphenyl)spiro[piperidine4,3'-pyrrolo[2,3-b]pyridin]-2'(1'H)-one (1). Compound 1 was obtained from tert-butyl 5'bromo-2'-oxo-1'-((2-

(trimethylsilyl)ethoxy)methyl)-1',2'-

dihydrospiro[piperidine-4,3'-pyrrolo[2,3-

b]pyridine]-1-carboxylate and

methoxyphenyl)boronic acid as an off-white solid . Yield: $120 \mathrm{mg}, 50 \%$, MP: $232-238^{\circ} \mathrm{C}$, Pale brown solid. FT- IR (KBr): vmax 3777, 3473, 2842, 1722, 1591, 1474, 1199, 1124, 1016, 756, $665 \mathrm{~cm}-1 .{ }^{1} \mathrm{H}-\mathrm{NMR}(400 \mathrm{MHz}$, $\left.\mathrm{CDCl}_{3}\right): \delta 11.07$ (brs, $\left.1 \mathrm{H}\right), 8.21(\mathrm{~s}, 1 \mathrm{H}), 7.88(\mathrm{~s}$, $1 \mathrm{H}), 7.39-7.35(\mathrm{~m}, 2 \mathrm{H}), 7.25-7.21(\mathrm{~m}, 1 \mathrm{H}), 3.96$ (s, 3H), 3.46-3.49 (m, 2H), 3.33-3.30 (m, 2H), 2.03-1.98 (m, 4H). ${ }^{13} \mathrm{C}$ NMR: $(100 \mathrm{MHz}$, DMSO-d 6 ): 28.52， 43.42， 55.52， 111.72, $120.90,126.47,127.94,129.21,130.22$, 131.91, 146.69, 154.76, 156.16, and 179.82 . LC-MS (ES-API): $m / z=310.3(\mathrm{M}+\mathrm{H})^{+}$.

4.2.2 5'-(3,4-Difluorophenyl)spiro[piperidine4,3'-pyrrolo[2,3-b]pyridin]-2'(1'H)-one

(2). 
Compound 2 was obtained from tert-butyl 5'bromo-2'-oxo-1'-((2-

(trimethylsilyl)ethoxy)methyl)-1',2'-

dihydrospiro[piperidine-4,3'-pyrrolo[2,3-

b]pyridine]-1-carboxylate and (3,4difluorophenyl)boronic acid as a white solid. Yield: $80 \mathrm{mg}, 65 \%$, MP: $253-255{ }^{\circ} \mathrm{C}$. FT-IR (KBr): vmax 3447, 3022, 2812, 1711, 1610, 1466, 1202, 1126, 836, $656 \mathrm{~cm}-1 .{ }^{1} \mathrm{H}-\mathrm{NMR}$ (400 MHz, DMSO-d 6 ): $\delta 8.28(\mathrm{~d}, J=2.0 \mathrm{~Hz}$, $1 \mathrm{H}), 7.88$ (d, $J=2.0 \mathrm{~Hz}, 1 \mathrm{H}), 7.43$ (d, $J=6.8$ $\mathrm{Hz}, 2 \mathrm{H}), 6.88(\mathrm{~d}, J=6.8 \mathrm{~Hz}, 2 \mathrm{H}), 3.82-3.75(\mathrm{~m}$, $2 \mathrm{H}), 3.45-3.39(\mathrm{~m}, 2 \mathrm{H}), 3.25-3.09(\mathrm{~m}, 4 \mathrm{H}) .{ }^{13} \mathrm{C}$ NMR: (100 MHz, DMSO-d6): 30.27, 40.67, 44.77, 116.96, 128.80, 129.02, 130.16, 130.70, 133.68, 145.76, 155.58, 158.78 and 181.64. LC-MS (ES-API): $m / z=296.3(\mathrm{M}+\mathrm{H})^{+}$.

4.2.3 3-(2'-Oxo-1',2'-dihydrospiro[piperidine4,3'-pyrrolo[2,3-b]pyridin]-5'-yl)benzonitrile (3). Compound $\mathbf{3}$ was obtained from tert-butyl 5'-bromo-2'-oxo-1'-((2-

(trimethylsilyl)ethoxy)methyl)-1',2'-

dihydrospiro[piperidine-4,3'-pyrrolo[2,3-

b]pyridine]-1-carboxylate and cyanophenyl)boronic acid as a white solid. Yield: $43 \mathrm{mg}, 36 \%$. MP: 201-208 ${ }^{\circ} \mathrm{C}$. FT-IR (KBr): vmax 3492, 3402, 3079, 2229, 1726, 1605, 1467, 1233, 1186, 1126, 794, 763, 531 cm-1. ${ }^{1} \mathrm{H}-\mathrm{NMR}$ (500 MHz, DMSO-d 6 ): $\delta 8.41$ $(\mathrm{d}, \mathrm{J}=2.0 \mathrm{~Hz}, 1 \mathrm{H}), 7.98(\mathrm{~d}, J=2.0 \mathrm{~Hz}, 1 \mathrm{H})$, 7.23-7.19 (m, 3H), $7.04(\mathrm{~d}, J=4.0 \mathrm{~Hz}, 2 \mathrm{H})$, $3.87(\mathrm{~s}, 3 \mathrm{H}), 3.79(\mathrm{~s}, 3 \mathrm{H}), 3.51-3.36(\mathrm{~m}, 4 \mathrm{H})$, 2.12-2.02 (m, 4H). ${ }^{13} \mathrm{CNMR}:(125 \mathrm{MHz}$, DMSO-d6): 28.42, 43.47, 55.59, 110.47, $112.25,118.84,127.33,129.57,130.14$, 130.41, 144.63, 148.57, 149.16, 154.86 and 179.76. LC-MS (ES-API): $m / z=305.3(\mathrm{M}+\mathrm{H})+$

4.2.4 5'-(4-Hydroxyphenyl)spiro[piperidine4,3'-pyrrolo[2,3-b]pyridin]-2'(1'H)-one Compound 4 was obtained from tert-butyl 5'bromo-2'-oxo-1'-((2-

(trimethylsilyl)ethoxy)methyl)-1',2'dihydrospiro[piperidine-4,3'-pyrrolo[2,3b]pyridine]-1-carboxylate and (4hydroxyphenyl)boronic acid as a white solid.
Yield: $40 \mathrm{mg}, 35 \%$. MP: $318-321{ }^{\circ} \mathrm{C}$, Brown solid. FT-IR (KBr): vmax 3447, 3022, 2812 , 1711, 1684, 1610, 1466, 1442, 1202, 1126, 836, 718, $658 \mathrm{~cm}-1 .{ }^{1} \mathrm{H}-\mathrm{NMR}\left(400 \mathrm{MHz}, \mathrm{CD}_{3} \mathrm{OD}\right)$ : $\delta 8.28(\mathrm{~d}, J=2.0 \mathrm{~Hz}, 1 \mathrm{H}), 7.88(\mathrm{~d}, J=2.0 \mathrm{~Hz}$, $1 \mathrm{H}), 7.45-7.41(\mathrm{~m}, 2 \mathrm{H}), 6.90-6.87(\mathrm{~m}, 2 \mathrm{H})$, 3.82-3.75 (m, 2H), 3.45-3.39 (m, 2H), 2.252.09 (m, 4H). ${ }^{13} \mathrm{C}$ NMR: (100 MHz, $\left.\mathrm{CD}_{3} \mathrm{OD}\right)$ : 30.27, 40.67, 44.77, 116.96, 128.80, 129.02, $130.16, \quad 130.70,133.68, \quad 145.76,155.58$, 158.78, and 181.64. LC-MS (ES-API): $m / z=$ $296.3(\mathrm{M}+\mathrm{H})^{+}$.

\subsection{5}

$5 '-(3,4-$

Dimethoxyphenyl)spiro[piperidine-4,3'-

pyrrolo[2,3-b]pyridin]-2'(1'H)-one (6) (Scheme 1): Compound 6 was obtained from tert-butyl 5'-bromo-2'-oxo-1'-((2-

(trimethylsilyl)ethoxy)methyl)-1',2'-

dihydrospiro[piperidine-4,3'-pyrrolo[2,3-

b]pyridine]-1-carboxylate and (3,4dimethoxyphenyl)boronic acid as a white solid. Yield: $150 \mathrm{mg}, 75 \%$, MP: $272-275^{\circ} \mathrm{C}$. FT-IR (KBr): vmax 3117, 2993, 2851, 1673, 1615, 1539, 1470, 1329, 1204, 1138, 838, 802, 721 cm-1. ${ }^{1} \mathrm{H}-\mathrm{NMR}$ (500 MHz, DMSO-d 6 ): $\delta 11.30$ (brs, $1 \mathrm{H}), 8.75$ (brs, 1H), $8.41(\mathrm{~d}, J=2.0 \mathrm{~Hz}$, $1 \mathrm{H}), 7.98(\mathrm{~d}, J=2.0 \mathrm{~Hz}, 1 \mathrm{H}), 7.23-7.19(\mathrm{~m}$, 2H), $7.04(\mathrm{~d}, J=8.5 \mathrm{~Hz}, 1 \mathrm{H}), 3.86(\mathrm{~s}, 3 \mathrm{H}), 3.79$ (s, 3H), 3.51-3.46 (m, 2H), 3.40-3.36 (m, 2H), 2.11-2.02 (m, 4H). ${ }^{13} \mathrm{C}$ NMR: $(125 \mathrm{MHz}$, DMSO-d $\left.{ }_{6}\right): \delta$ 28.42, 43.47, 55.44, 110.47, $112.25, \quad 118.84,127.33,129.57,130.14$, $130.41,144.63,148.57,149.16,154.86$, and 179.76. LC-MS (ES-API): $m / z=340.0(\mathrm{M}+\mathrm{H})^{+}$.

4.2.6. 5'-(2,6-Difluorophenyl)spiro[piperidine4,3'-pyrrolo[2,3-b]pyridin]-2'(1'H)-one (Scheme 1): Compound 8 was obtained from tert-butyl 5'-bromo-2'-oxo-1'-((2(trimethylsilyl)ethoxy)methyl)-1',2'-

dihydrospiro[piperidine-4,3'-pyrrolo[2,3-

b]pyridine]-1-carboxylate and (2,6difluorophenyl)boronic acid as a white solid. Yield: $75.0 \mathrm{mg}, 61 \%$, MP: $280-284{ }^{\circ} \mathrm{C}$. White solid. FT-IR (KBr): vmax 3094, 1730, 1602, 1467, 1321, 1223, 772, $669 \mathrm{~cm}-1 .{ }^{1} \mathrm{H}-\mathrm{NMR}$ 
(500 MHz, DMSO-d 6$): \delta 11.38(\mathrm{~s}, 1 \mathrm{H}), 8.86(\mathrm{~s}$, $1 \mathrm{H}), 8.46(\mathrm{~s}, 1 \mathrm{H}), 8.10(\mathrm{~s}, 1 \mathrm{H}), 7.87(\mathrm{t}, J=10.0$ $\mathrm{Hz}, 1 \mathrm{H})$, 7.57-7.51 (m, 2H), 3.52-3.41 (m, 4H), 2.11-1.98 (m, 4H). ${ }^{13} \mathrm{C}$ NMR: $(125 \mathrm{MHz}$, DMSO-d 6$): \delta$ 28.77, 44.18, 116.18, 116.32, $118.36,118.49, \quad 123.91,127.80,128.67$, 130.27, 135.61, 145.70, 156.28, and 180.15. LC-MS (ES-API): $m / z=316.1(\mathrm{M}+\mathrm{H})^{+}$.

4.3 3-(2'-Oxo-1',2'-dihydrospiro[piperidine4,3'-pyrrolo[2,3-b]pyridin]-5'-yl)acrylic acid (5): A solution mixture of arylbromo tert-butyl 5'-bromo-2'-oxo-1'-((2-

(trimethylsilyl)ethoxy)methyl)-1',2'dihydrospiro[piperidine-4,3'-pyrrolo[2,3-

b]pyridine]-1-carboxylate I (1.0 eq, 0.15 mmol), $t$-butylacrylate (1.5 eq.), $\mathrm{PdCl}_{2}\left(\mathrm{PPh}_{3}\right)_{2}$ (0.05 eq), triethylamine (2.0 eq) in DMF (5.0 $\mathrm{mL}$ ) were stirred at $100{ }^{\circ} \mathrm{C}$ for $1 \mathrm{~h}$ under $\mathrm{MW}$ irradiation. The reaction mixture was diluted with water, extracted with ethyl acetate twice. The combined extracts were washed with brine, dried over anhydrous $\mathrm{Na}_{2} \mathrm{SO}_{4}$ and concentrated. The crude was purified by column chromatography over silica gel (100-200 mesh) using a solvent gradient mixture of $40 \%$ ethyl acetate in pet-ether as an eluent afforded desired compound. This compound $(0.1 \mathrm{mmol})$ on de-protection with TFA $(2.0 \mathrm{~mL})$ in dichloromethane $(2.0 \mathrm{~mL})$ at $60^{\circ} \mathrm{C}$ for $10 \mathrm{~h}$. Distilled off the volatiles under reduced pressure. The crude residue was purified by GRACE flash chromatography using C18 column with $0.1 \%$ formic acid in water and acetonitrile as an eluent to obtain the title compound. Yield 64\%, MP: $280-284{ }^{\circ} \mathrm{C}$. White solid. FT-IR (KBr): vmax 3217, 3015, 2824, 1726, 1675, 1624, 1481, 1404, 1212, 1139, 976, 720, $666 \mathrm{~cm}-1 .{ }^{1} \mathrm{H}-\mathrm{NMR}$ (500 MHz, DMSO$\left.\mathrm{d}_{6}\right): \delta 12.39$ (brs, $\left.1 \mathrm{H}\right), 11.50(\mathrm{~s}, 1 \mathrm{H}), 8.71$ (brs, $1 \mathrm{H}), 8.40(\mathrm{~s}, 1 \mathrm{H}), 8.23(\mathrm{~s}, 1 \mathrm{H}), 7.61(\mathrm{~d}, J=16.0$ $\mathrm{Hz}, 1 \mathrm{H}), 7.65(\mathrm{~d}, J=16.0 \mathrm{~Hz}, 1 \mathrm{H}), 3.48-3.36$ (m, 4H), 2.08-1.93 (m, 4H). ${ }^{13} \mathrm{C}$ NMR: (125 MHz, DMSO-d6): $\delta$ 28.21, 39.16 (merged in DMSO signal), 43.63, 118.43, 124.75, 127.64, $128.96,141.07,148.86,157.39,167.58$, and
179.59. LC-MS (ES-API): $\mathrm{m} / \mathrm{z}=274.0$ $(\mathrm{M}+\mathrm{H})^{+}$.

4.4 5'-(3,4-Dihydroxyphenyl)spiro[piperidine4,3'-pyrrolo[2,3-b]pyridin]-2'(1'H)-one (7): To a solution of $6(250 \mathrm{mg})$ in dichloromethane (10 $\mathrm{mL}$ ) was cooled to $0^{\circ} \mathrm{C}$ and added a solution of $\mathrm{BBr}_{3}$ (5.0 eq.) (1.0 $\mathrm{M}$ in DCM) drop wise. The resulting reaction mixture was stirred at ambient temperature for $16 \mathrm{~h}$ under $\mathrm{N}_{2}$. The resulting mixture was quenched with methanol $(2.0 \mathrm{~mL})$ and concentrated. The residue was alkaline with $\mathrm{NaHCO}_{3}$ solution, extracted with ethyl acetate $(30 \mathrm{~mL} \times 3)$. The combined organic phase was washed with brine $(50 \mathrm{~mL})$, dried over anhydrous $\mathrm{Na}_{2} \mathrm{SO}_{4}$ and concentrated under reduced pressure to give a residue, which was purified by GRACE flash chromatography using $\mathrm{C} 18$ column with $0.1 \%$ formic acid in water and acetonitrile as an eluent to obtain the title compound as a brown solid. Yield: $50 \%$, M.P: $255-258^{\circ} \mathrm{C}$. FT-IR (KBr): vmax 3094, 1703, 1602, 1467, 1321, 1223, 772, $669 \mathrm{~cm}-1$. ${ }^{1} \mathrm{H}-\mathrm{NMR}\left(500 \mathrm{MHz}, \mathrm{DMSO}-\mathrm{d}_{6}\right): \delta 11.13$ (s, $1 \mathrm{H}), 8.39(\mathrm{~s}, 1 \mathrm{H}), 8.24(\mathrm{~s}, 1 \mathrm{H}), 7.91(\mathrm{~s}, 1 \mathrm{H})$, $7.04(\mathrm{~s}, 1 \mathrm{H}), 6.92(\mathrm{~s}, 1 \mathrm{H}), 6.81(\mathrm{~s}, 1 \mathrm{H}), 3.40-$ 3.21 (m, 4H), 1.91-1.83 (m, 4H). ${ }^{13} \mathrm{C}$ NMR: (125 MHz, DMSO-d 6 ): $\delta 30.25,44.93,75.91$, $114.50, \quad 116.68, \quad 118.01, \quad 128.47,129.16$, 129.66, 131.10, 144.29, 145.85, 146.37, 154.99, and 180.60. LC-MS (ES-API): $\mathrm{m} / \mathrm{z}=$ $312.0(\mathrm{M}+\mathrm{H})+$.

\subsection{Synthesis of compounds 9 to 18 (Following general method B)}

4.5.1 5'-(Benzo[d][1,3]dioxol-5-yl)-1-(1methyl-1H-1,2,3-triazole-4-

carbonyl)spiro[piperidine-4,3'-pyrrolo[2,3-

b]pyridin]-2'(1'H)-one (9): Compound 9 was obtained from 5'-(benzo[d][1,3]dioxol-5yl)spiro[piperidine-4,3'-pyrrolo[2,3-b]pyridin]2'(1'H)-one and 1-methyl-1H-1,2,3-triazole-4carboxylic acid as a white solid. Yield: $55 \mathrm{mg}$, 41\%, MP: $215-217{ }^{\circ} \mathrm{C}$. FT-IR (KBr): vmax 3421, 3099, 1716, 1643, 1465, 1213, 1033, 806, $669 \mathrm{~cm}^{-1}$. ${ }^{1} \mathrm{H}-\mathrm{NMR}$ (400 MHz, DMSO-d 6 ): $\delta$ 
$8.51(\mathrm{~s}, 1 \mathrm{H}), 8.35$ (d, $J=2.0 \mathrm{~Hz}, 1 \mathrm{H}), 8.17(\mathrm{~d}, J$ $=2.0 \mathrm{~Hz}, 1 \mathrm{H}), 7.34(\mathrm{~d}, J=2.4 \mathrm{~Hz}, 1 \mathrm{H}), 7.19$ $(\mathrm{dd}, J=1.6,8.0 \mathrm{~Hz}, 1 \mathrm{H}), 6.98(\mathrm{~d}, J=8.0 \mathrm{~Hz}$, $1 \mathrm{H}), 6.05$ (s, 2H), 4.40-4.33 (m, 2H), $4.10(\mathrm{~s}$, $3 \mathrm{H}), 4.03-3.98(\mathrm{~m}, 2 \mathrm{H}), 1.91-1.86(\mathrm{~m}, 4 \mathrm{H}) .{ }^{13} \mathrm{C}$ NMR: (100 MHz, DMSO-d $): \delta 31.93,32.86$, $36.93,38.09,42.22,45.90,101.60,107.59$, $109.11, \quad 120.56, \quad 128.73, \quad 129.60,130.15$, $130.43, \quad 132.07,143.58,144.60,147.25$, 148.46, 155.45, 160.28, and 180.87. LC-MS $(\mathrm{ES}-\mathrm{API}): \mathrm{m} / \mathrm{z}=433.1(\mathrm{M}+\mathrm{H})^{+}$.

4.5.2 5'-(Benzo[d][1,3]dioxol-5-yl)-1-(1methyl-1H-pyrazole-5-

carbonyl)spiro[piperidine-4,3'-pyrrolo[2,3b]pyridin]-2'(1'H)-one (10): Compound $\mathbf{1 0}$ was obtained from 5'-(benzo[d][1,3]dioxol-5yl)spiro[piperidine-4,3'-pyrrolo[2,3-b]pyridin]$2^{\prime}(1 ' \mathrm{H})$-one and 1-fluorocyclopropane-1carboxylic acid as a white solid. Yield: $60 \mathrm{mg}$, 34\%. MP: $312-315{ }^{\circ} \mathrm{C}$. FT-IR (KBr): vmax 3412, 2935, 2711, 1706, 1633, 1458, 1230, 1031, 786, $669 \mathrm{~cm}^{-1}$. ${ }^{1} \mathrm{H}-\mathrm{NMR}(500 \mathrm{MHz}$, DMSO-d 6 ): $\delta 11.16(\mathrm{~s}, 1 \mathrm{H}), 8.34(\mathrm{~s}, 1 \mathrm{H}), 8.15$ (s, 1H), $7.48(\mathrm{~s}, 1 \mathrm{H}), 7.32(\mathrm{~s}, 1 \mathrm{H}), 7.18(\mathrm{~d}, J=$ $7.5 \mathrm{~Hz}, 1 \mathrm{H}), 7.00(\mathrm{~d}, J=7.5 \mathrm{~Hz}, 1 \mathrm{H}), 6.53(\mathrm{~s}$, $1 \mathrm{H}), 6.06(\mathrm{~s}, 2 \mathrm{H}), 4.10-3.75(\mathrm{~m}, 7 \mathrm{H}), 1.95-1.80$ $(\mathrm{m}, 4 \mathrm{H}),{ }^{13} \mathrm{C}$ NMR: (125 MHz, DMSO-d 6$): \delta$ $31.31,37.60,42.52,45.23,101.14,106.24$, $107.09, \quad 108.66,120.09, \quad 128.03,129.73$, 129.99, 131.61, 135.47, 137.38, 144.19, 146.79, 147.97, 154.96, 160.47 and 180.28. LC-MS (ES-API): $m / z=432.1(\mathrm{M}+\mathrm{H})^{+}$.

4.5.3. 5 '-(Benzo[d] $[1,3]$ dioxol-5-yl)-1-(1fluorocyclopropane-1-

carbonyl)spiro[piperidine-4,3'-pyrrolo[2,3-

b]pyridin]-2'(1'H)-one (11): Compound 11 was obtained from 5'-(benzo[d][1,3]dioxol-5yl)spiro[piperidine-4,3'-pyrrolo[2,3-b]pyridin]2'(1'H)-one and 1-methyl-1H-pyrazole-5carboxylic acid as an off-white white solid. Yield: $60 \mathrm{mg}, 47 \%$, MP: $235-240{ }^{\circ} \mathrm{C}$. FT-IR (KBr): vmax 3428, 3097, 1715, 1645, 1469, 1214, 1305, 802, 808, 671, $518 \mathrm{~cm}^{-1}$. ${ }^{1} \mathrm{H}-\mathrm{NMR}$ (500 MHz, DMSO-d 6$): \delta 11.17(\mathrm{~s}, 1 \mathrm{H}), 8.35(\mathrm{~s}$, $1 \mathrm{H}), 8.16$ (s, 1H), 7.33 (s, 1H), 7.19 (d, $J=7.5$
$\mathrm{Hz}, 1 \mathrm{H}), 6.99$ (d, $J=8.0 \mathrm{~Hz}, 1 \mathrm{H}), 6.05(\mathrm{~s}, 2 \mathrm{H})$, 4.10-3.80 (m, 4H), 1.96-1.78 (m, 4H), 1.31$1.22(\mathrm{~m}, 4 \mathrm{H}) .{ }^{1} \mathrm{H}-\mathrm{NMR}\left(400 \mathrm{MHz}, \mathrm{CDCl}_{3}\right): \delta$ $8.47(\mathrm{~s}, 1 \mathrm{H}), 8.30(\mathrm{~s}, 1 \mathrm{H}), 7.64(\mathrm{~s}, 1 \mathrm{H}), 6.98-$ $6.96(\mathrm{~m}, 2 \mathrm{H}), 6.90(\mathrm{~d}, J=8.0 \mathrm{~Hz}, 1 \mathrm{H}), 6.02(\mathrm{~s}$, $2 \mathrm{H}), 4.22-4.89(\mathrm{~m}, 4 \mathrm{H}), 2.05-1.94(\mathrm{~m}, 4 \mathrm{H})$, 1.40-1.25 (m, 4H). ${ }^{13} \mathrm{C}$ NMR: $(125 \mathrm{MHz}$, DMSO-d 6 ): $\delta$ 10.72, 45.29, 77.34, 79.18, $101.12, \quad 107.07, \quad 108.63, \quad 120.07, \quad 128.15$, $129.65, \quad 129.96, \quad 131.57,144.14,146.76$, 147.97, 154.95, 164.99, 165.14, and 180.34. LC-MS (ES-API): $m / z=410.1(\mathrm{M}+\mathrm{H})^{+}$.

4.5.4 5'-(Benzo[d][1,3]dioxol-5-yl)-1-(2fluoro-2-methylpropanoyl)spiro[piperidine4,3'-pyrrolo[2,3-b]pyridin]-2'(1'H)-one (12): Compound 12 was obtained from 5'(benzo[d][1,3]dioxol-5-yl)spiro[piperidine-

4,3'-pyrrolo[2,3-b]pyridin]-2'(1'H)-one and 2fluoro-2-methylpropanoic acid a white solid. Yield: $55 \mathrm{mg}, 44 \%$. MP: 249-253 ${ }^{\circ} \mathrm{C}$. FT-IR (KBr): vmax 3425, 3089, 2985, 1722, 1629, 1458, 1217, 1031, 806, $655 \mathrm{~cm}^{-1} .{ }^{1} \mathrm{H}-\mathrm{NMR}$ (500 MHz, DMSO-d $\left.\mathrm{d}_{6}\right): \delta 11.16(\mathrm{~s}, 1 \mathrm{H}), 8.34(\mathrm{~d}, J=$ $1.5 \mathrm{~Hz}, 1 \mathrm{H}), 8.21$ (brs, $1 \mathrm{H}), 7.63$ (d, $J=2.0 \mathrm{~Hz}$, $1 \mathrm{H}), 6.98-6.96(\mathrm{~m}, 2 \mathrm{H}), 6.90(\mathrm{~d}, J=8.0 \mathrm{~Hz}$, $1 \mathrm{H}), 6.02(\mathrm{~s}, 2 \mathrm{H}), 4.22-4.18(\mathrm{~m}, 3 \mathrm{H}), 3.91-3.89$ $(\mathrm{m}, 1 \mathrm{H}), 2.03-1.99(\mathrm{~m}, 2 \mathrm{H}), 1.94-1.88(\mathrm{~m}, 2 \mathrm{H})$, 1.69 (s, 3H), 1.65 (s, 3H). ${ }^{1} \mathrm{H}-\mathrm{NMR}(500 \mathrm{MHz}$, $\left.\mathrm{CDCl}_{3}\right): \delta 8.30(\mathrm{~d}, J=2.0 \mathrm{~Hz}, 1 \mathrm{H}), 8.21(\mathrm{~s}, 1 \mathrm{H})$, $7.63(\mathrm{~d}, J=2.0 \mathrm{~Hz}, 1 \mathrm{H}), 6.98-6.96(\mathrm{~m}, 3 \mathrm{H})$, $6.90(\mathrm{~d}, J=8.0 \mathrm{~Hz}, 1 \mathrm{H}), 6.02(\mathrm{~s}, 2 \mathrm{H}), 4.19-4.18$ (m, 3H), 3.91-3.89 (m, 1H), 2.03-1.99 (m, 2H), 1.93-1.90 (m, 2H), 1.67 (d, $J=21.5 \mathrm{~Hz}, 6 \mathrm{H})$. ${ }^{13} \mathrm{C}$ NMR: (125 MHz, DMSO-d 6 ): 25.66, 25.85, 45.31, 95.91, 97.34, 101.11, 107.12, 108.61, $120.10, \quad 128.18, \quad 129.66,129.94,131.57$, $144.12,146.75,147.95,154.94,169.19$ and 180.35. LC-MS (ES-API): $m / z=412.1$ $(\mathrm{M}+\mathrm{H})^{+}$.

4.5.5 (3aS,4S,6aR)-4-(5-Oxo-5-(2'-оxo-5'(3,4,5-trimethoxyphenyl)-1',2'-

dihydrospiro[piperidine-4,3'-pyrrolo[2,3b]pyridin]-1-yl)pentyl)tetrahydro- $1 \mathrm{H}$ thieno[3,4-d]imidazol-2(3H)-one (13): A solution of 5 '- $(3,4,5-$ 
trimethoxyphenyl)spiro[piperidine-4,3'-

pyrrolo[2,3-b]pyridin]-2'(1'H)-one III (0.271

mmol), 2,5-dioxopyrrolidin-1-yl 5((3aS,4S,6aR)-2-oxohexahydro-1H-thieno[3,4d]imidazol-4-yl)pentanoate $(0.271 \mathrm{mmol})$ and triethylamine $(0.541 \mathrm{mmol})$ in DMF $(2.0 \mathrm{~mL})$ were stirred at room temperature for $16 \mathrm{~h}$. The reaction mixture was partitioned between ethyl acetate $(30 \mathrm{ml} \times 2)$ and water $(50 \mathrm{~mL})$. The separated organic layer was washed with brine, dried over anhyd. $\mathrm{Na}_{2} \mathrm{SO}_{4}$ and concentrated. The crude was purified by GRACE flash chromatography using C18 column with $0.1 \%$ formic acid in water and acetonitrile as an eluent afforded the title compound as a white solid. Yield: $62 \mathrm{mg}$, $46 \%$, M.P: $242-246^{\circ} \mathrm{C}$. FTIR (KBr): vmax 3404, 2927, 1712, 1598, 1458, 1230, 1116, 1012, 839, $667 \mathrm{~cm}^{-1} .{ }^{1} \mathrm{H}-\mathrm{NMR}(500$ MHz, DMSO-d 6$): \delta 11.12(\mathrm{~s}, 1 \mathrm{H}), 8.41(\mathrm{~d}, J=$ $1.5 \mathrm{~Hz}, 1 \mathrm{H}), 8.10(\mathrm{~s}, 1 \mathrm{H}), 6.90(\mathrm{~s}, 2 \mathrm{H}), 6.45(\mathrm{~s}$, $1 \mathrm{H}), 6.36(\mathrm{~s}, 1 \mathrm{H}), 4.31-4.29(\mathrm{~m}, 1 \mathrm{H}), 4.15-4.13$ $(\mathrm{m}, 1 \mathrm{H}), 4.01-3.68(\mathrm{~m}, 13 \mathrm{H}), 3.14-3.12(\mathrm{~m}$, $1 \mathrm{H})$, 2.84-2.78 $(\mathrm{m}, 1 \mathrm{H}), 2.58-2.56(\mathrm{~m}, 1 \mathrm{H})$, 2.48-2.32 (m, $2 \mathrm{H}), 1.91-1.72(\mathrm{~m}, 4 \mathrm{H}), 1.68-$ $1.47(\mathrm{~m}, 4 \mathrm{H}), 1.40-1.36(\mathrm{~m}, 2 \mathrm{H}) .{ }^{13} \mathrm{C}$ NMR: (125 MHz, DMSO-d 6 ): 24.93, 28.16, 28.39, 31.47, 32.24, 36.44, 45.21, 55.50, 56.07, 59.17, $60.05,61.05,104.22,128.30,129.75,130.49$, $133.30, \quad 137.08, \quad 144.68, \quad 153.25, \quad 155.24$, 162.70, 170.61 and 180.44. LC-MS (ES-API): $\mathrm{m} / \mathrm{z}=596.2(\mathrm{M}+\mathrm{H})+$.

4.5.6.

(3aS, 4S,6aR)-4-(5-(5'(Benzo[d][1,3]dioxol-5-yl)-2'-oxo-1',2'dihydrospiro[piperidine-4,3'-pyrrolo[2,3b]pyridin]-1-yl)-5-oxopentyl)tetrahydro-1Hthieno[3,4-d]imidazol-2(3H)-one (14)

Compound 14 was prepared by using the same procedure of compound $\mathbf{1 3}$ from 5'(benzo[d][1,3]dioxol-5-yl)spiro[piperidine4,3'-pyrrolo[2,3-b]pyridin]-2'(1'H)-one III and 2,5-dioxopyrrolidin-1-yl 5-((3aS,4S,6aR)-2oxohexahydro-1H-thieno[3,4-d]imidazol-4yl)pentanoate as a white solid. Yield: $68 \mathrm{mg}$, $51 \%$, M.P: $185-187^{\circ} \mathrm{C}$. FT-IR (KBr): vmax 3435, 3273, 2922, 1712, 1618, 1456, 1222, 1026, 931, 794, $675 \mathrm{~cm}^{-1} .{ }^{1} \mathrm{H}-\mathrm{NMR}(400 \mathrm{MHz}$,
DMSO-d $\left.\mathrm{d}_{6}\right): \delta 11.12(\mathrm{~s}, 1 \mathrm{H}), 8.34(\mathrm{~s}, 1 \mathrm{H}), 8.10$ (s, $1 \mathrm{H}), 7.32-6.98(\mathrm{~m}, 3 \mathrm{H}), 6.45-6.32(\mathrm{~m}, 2 \mathrm{H})$, $6.05(\mathrm{~s}, 2 \mathrm{H}), 4.30-4.15(\mathrm{~m}, 2 \mathrm{H}), 3.90-3.79(\mathrm{~m}$, $4 \mathrm{H}), 3.12(\mathrm{~s}, 1 \mathrm{H}), 2.83-2.81(\mathrm{~m}, 1 \mathrm{H}), 2.59-2.37$ (m, 3H), 1.83-1.39 (m, 10H). ${ }^{13} \mathrm{C}$ NMR: $(125$ MHz, DMSO-d 6 ): 24.95, 28.14, 28.38, 31.45, 32.12, 32.21, 36.52, 45.34, 55.49, 59.16, 61.03, $101.12, \quad 107-08, \quad 108.64, \quad 120.06, \quad 128.28$, $129.60,129.91,131.60, \quad 144.09, \quad 146.76$, 147.97, 154.97, 162.68, 170.64 and 180.41. LC-MS (ES-API): $m / z=550.1(\mathrm{M}+\mathrm{H})^{+}$.

4.5.7. Methyl 1-(5'-(benzo[d][1,3]dioxol-5-yl)2'-oxo-1',2'-dihydrospiro[piperidine-4,3'-

pyrrolo[2,3-b]pyridine]-1-

carbonyl)cyclopropane-1-carboxylate (15): Compound $\mathbf{1 5}$ was obtained from 5'(benzo[d][1,3]dioxol-5-yl)spiro[piperidine4,3'-pyrrolo[2,3-b]pyridin]-2'(1'H)-one and 1(methoxycarbonyl)cyclopropane-1-carboxylic acid as a pale yellow solid. Yield: $68 \mathrm{mg}, 49 \%$. M.P: $280-284^{\circ} \mathrm{C}$. FT-IR (KBr): vmax 3425 , 3099, 2956, 1722, 1633, 1458, 1222, 1149, 1029, 837, $659 \mathrm{~cm}^{-1}$. ${ }^{1} \mathrm{H}-\mathrm{NMR}(500 \mathrm{MHz}$, $\left.\mathrm{CDCl}_{3}\right): \delta 8.45($ brs, $1 \mathrm{H}), 8.30(\mathrm{~d}, J=2.0 \mathrm{~Hz}$, $1 \mathrm{H}), 7.62(\mathrm{~d}, J=2.0 \mathrm{~Hz}, 1 \mathrm{H}), 7.97-7.95(\mathrm{~m}$, $2 \mathrm{H}), 6.91(\mathrm{~d}, J=8.0 \mathrm{~Hz}, 1 \mathrm{H}), 6.02(\mathrm{~s}, 2 \mathrm{H}), 4.20-$ $4.17(\mathrm{~m}, 1 \mathrm{H}), 4.05-4.00(\mathrm{~m}, 1 \mathrm{H}), 3.92-3.91(\mathrm{~m}$, $1 \mathrm{H}), 3.82-3.78(\mathrm{~m}, 1 \mathrm{H}), 3.76(\mathrm{~s}, 3 \mathrm{H}), 2.02-1.88$ (m, 4H), 1.58-1.52 (m, 2H), 1.43-1.38 (m, 2H). ${ }^{13}$ C NMR: (100 MHz, DMSO-d 6 ): $15.31,15.53$, $28.21,31.20,31.35,37.06,45.15,52.45$, $101.13, \quad 107.05, \quad 108.66, \quad 120.05, \quad 128.17$, $129.55, \quad 129.96, \quad 131.62, \quad 144.16, \quad 146.77$, 147.97, 154.96, 165.44, 171.52 and 180.34 . LC-MS (ES-API): $m / z=450.1(\mathrm{M}+\mathrm{H})^{+}$.

4.5.8. 5'-(Benzo[d][1,3]dioxol-5-yl)-1-(3methyloxetane-3-carbonyl)spiro[piperidine4,3'-pyrrolo[2,3-b]pyridin]-2'(1'H)-one (16): Compound 16 was obtained from 5'(benzo[d][1,3]dioxol-5-yl)spiro[piperidine4,3'-pyrrolo[2,3-b]pyridin]-2'(1'H)-one and 3methyloxetane-3-carboxylic acid as an offwhite solid. Yield: $48 \mathrm{mg}, 37 \%$. M.P: 281$285^{\circ} \mathrm{C}$. FT-IR (KBr): vmax 3427, 2877, 1722 , $1624,1456,1219,1029,829,657 \mathrm{~cm}^{-1} .{ }^{1} \mathrm{H}-$ 
NMR (400 MHz, DMSO-d 6 ): $\delta 11.14(\mathrm{~s}, 1 \mathrm{H})$, $8.37(\mathrm{~s}, 1 \mathrm{H}), 8.17(\mathrm{~s}, 1 \mathrm{H}), 7.32(\mathrm{~s}, 1 \mathrm{H}), 7.17(\mathrm{~d}$, $J=8.0 \mathrm{~Hz}, 2 \mathrm{H}), 7.00(\mathrm{~d}, J=8.0 \mathrm{~Hz}, 1 \mathrm{H}), 6.06$ $(\mathrm{s}, 2 \mathrm{H}), 4.87(\mathrm{dd}, J=5.6,16.8 \mathrm{~Hz}, 2 \mathrm{H}), 4.32-$ $4.28(\mathrm{~m}, 2 \mathrm{H}), 3.93-3.20(\mathrm{~m}, 6 \mathrm{H}), 1.95-75(\mathrm{~m}$, $4 \mathrm{H}), 1.59$ (s, 3H). ${ }^{13} \mathrm{C}$ NMR (100 MHz, DMSO$\left.\mathrm{d}_{6}\right): \delta 23.00,31.39,31.73,36.59,43.95,45.17$, 78.27, 78.75, 101.12, 107.05, 108.64, 120.06, $128.08, \quad 129.72, \quad 129.93, \quad 131.59, \quad 144.12$, 146.76, 147.96, 154.94, 172.32, and 180.30. LC-MS (ES-API): $m / z=422.1(\mathrm{M}+\mathrm{H})^{+}$.

4.5.9. Methyl 1-(2'-oxo-5'-(3,4,5trimethoxyphenyl)-1',2'-

dihydrospiro[piperidine-4,3'-pyrrolo[2,3-

b]pyridine]-1-carbonyl)cyclopropane-1-

carboxylate (17): Compound 17 was obtained from 5'-(3,4,5-

trimethoxyphenyl)spiro[piperidine-4,3'-

pyrrolo[2,3-b]pyridin]-2'(1'H)-one and 1(methoxycarbonyl)cyclopropane-1-carboxylic acid as a white solid. Yield: $70 \mathrm{mg}, 52 \%$. MP: 240-242 ${ }^{\circ} \mathrm{C}$. FT-IR (KBr): vmax 3439, 3236, 2939, 1724, 1633, 1456, 1220, 1130, 1016, 837, $657,557 \mathrm{~cm}^{-1} .{ }^{1} \mathrm{H}-\mathrm{NMR}$ (500 MHz, DMSO-d 6$)$ : $\delta 11.17(\mathrm{~s}, 1 \mathrm{H}), 8.40(\mathrm{~d}, J=2.0 \mathrm{~Hz}, 1 \mathrm{H}), 8.04$ $(\mathrm{d}, J=1.5 \mathrm{~Hz}, 1 \mathrm{H}), 6.90(\mathrm{~s}, 2 \mathrm{H}), 4.04-4.01(\mathrm{~m}$, $1 \mathrm{H}), 3.92-3.67(\mathrm{~m}, 15 \mathrm{H}), 1.97-1.79(\mathrm{~m}, 4 \mathrm{H})$, 1.45-1.33 (m, 4H). ${ }^{13} \mathrm{C}$ NMR: (125 MHz, DMSO-d 6 ): 15.40, 15.52, 28.21, 31.15, 31.49, $37.02,40.91,45.02,52.43,56.05,60.03$, $104.29,128.13,129.71,130.54,1333.32$, $137.10, \quad 144.73, \quad 153.24, \quad 155.20,165.48$, 171.52, and 180.35. LC-MS (ES-API): $\mathrm{m} / \mathrm{z}=$ $496.1(\mathrm{M}+\mathrm{H})^{+}$.

4.5.10. 5'-(Benzo[d][1,3]dioxol-5-yl)-1-(4methylmorpholine-3-

carbonyl)spiro[piperidine-4,3'-pyrrolo[2,3-

b]pyridin]-2'(1'H)-one (18): Compound 18 was obtained 5'-(3,4,5trimethoxyphenyl)spiro[piperidine-4,3'-

pyrrolo[2,3-b]pyridin]-2'(1'H)-one and 4methylmorpholine-3-carboxylic acid as a white solid. Yield: $72 \mathrm{mg}, 52 \% \mathrm{MP}$ : 287-290 ${ }^{\circ} \mathrm{C}$. White solid. FT-IR (KBr): vmax 3423, 3172, 1707, 1653, 1467, 1386, 1232, 1039, 842, 557 $\mathrm{cm}^{-1} .{ }^{1} \mathrm{H}-\mathrm{NMR}\left(400 \mathrm{MHz}, \mathrm{CDCl}_{3}\right): \delta 11.18$ (bs, $1 \mathrm{H}), 8.35(\mathrm{~s}, 1 \mathrm{H}), 8.13(\mathrm{~s}, 1 \mathrm{H}), 7.31(\mathrm{~s}, 1 \mathrm{H})$, $7.16(\mathrm{t}, J=5.5 \mathrm{~Hz}, 1 \mathrm{H}), 7.0(\mathrm{~d}, J=8.5 \mathrm{~Hz}, 1 \mathrm{H})$, $6.06(\mathrm{~s}, 2 \mathrm{H}), 4.18-3.49(\mathrm{~m}, 9 \mathrm{H}), 3.07-2.61(\mathrm{~m}$, 4H), 1.93-1.78 (m, 4H). ${ }^{13} \mathrm{C}$ NMR: (125 MHz, DMSO-d $)_{6}: \delta 31.26,32.02,32.38,37.96,40.83$, 44.93, 52.87, 66.01, 101.17, 107.14, 108.69, $120.16, \quad 127.97, \quad 129.53,131.62,144.30$, 146.81, 147.97, 154.99, 163.02, and 180.14. LC-MS (ES-API): $m / z=451.1(\mathrm{M}+\mathrm{H})^{+}$.

4.5.11. 5 '-(Benzo[d][1,3]dioxol-5-yl)-1-(1(fluoromethyl)cyclopropane-1-

carbonyl)spiro[piperidine-4,3'-pyrrolo[2,3-

b]pyridin]-2'(1'H)-one (19): Compound 19 was obtained 5'-(3,4,5trimethoxyphenyl)spiro[piperidine-4,3'-

pyrrolo[2,3-b]pyridin]-2'(1'H)-one and 1(fluoromethyl)cyclopropane-1-carboxylic acid as an off-white solid. Yield: $52 \mathrm{mg}, 40 \%$. MP: 259-263 ${ }^{\circ} \mathrm{C}$. FT-IR (KBr): vmax 3427, 2914, 1720, 1631, 1465, 1226, 1035, 933, 806, 669 $\mathrm{cm}^{-1} .{ }^{1} \mathrm{H}-\mathrm{NMR}\left(500 \mathrm{MHz}, \mathrm{DMSO}-\mathrm{d}_{6}\right): \delta 11.15$ $(\mathrm{s}, 1 \mathrm{H}), 8.34(\mathrm{~s}, 1 \mathrm{H}), 8.13(\mathrm{~s}, 1 \mathrm{H}), 7.32(\mathrm{~s}, 1 \mathrm{H})$, $7.17(\mathrm{~d}, J=7.5 \mathrm{~Hz}, 1 \mathrm{H}), 6.99(\mathrm{~d}, J=8.0 \mathrm{~Hz}$, $1 \mathrm{H}), 6.05(\mathrm{~s}, 2 \mathrm{H}), 4.47(\mathrm{~d}, J=48.5 \mathrm{~Hz}, 2 \mathrm{H})$, 3.90 (brs, 4H), 1.79 (brs, 4H), 1.03 (s, 2H), 0.90 (s, 2H). ${ }^{13} \mathrm{C}$ NMR: (125 MHz, DMSO-d 6 ): $\delta$ 10.17, 25.26, 25.46, 31.62, 38.26, 45.34, 87.77, 89.12, 101.13, 107.09, 108.64, 120.10, 128.19, $129.70, \quad 129.93,131.62,144.11,146.76$, 147.96, 154.98, 168.81 and 180.36. LC-MS (ES-API): $m / z=424.1(\mathrm{M}+\mathrm{H})^{+}$.

\subsection{Cell lines}

RAMOS, A549, HCT116 (parental and p53 $3^{-/}$), CCRF-CEM, K562, U2OS, BJ and MRC-5 cell lines were obtained from ATCC (Middlesex, UK) and maintained according to recommendations. Multidrug-resistant sublines (CEM-DNR, K562-TAX) expressing the LRP and P-glycoprotein transporter proteins were derived and cultured as previously described [36]. Jurkat cells were purchased from DSMZ (Braunschweig, Germany) and maintained according to recommendations. All cell lines 
were maintained in a standard $5 \% \mathrm{CO} 2 /$ atmospheric air humidified incubator at $37^{\circ} \mathrm{C}$. Cell lines were routinely tested for mycoplasma contamination and authenticated biweekly or monthly.

\subsection{Cytotoxicity assay}

The cytotoxicity activity of all 19 compounds was tested under in vitro conditions using a 3-

\section{Authors declaration}

The authors declare no conflicting interests.

\section{Acknowledgements}

RG acknowledges DST-SERB (ECR/2016/000288), India for providing financial assistance, and GITAM for providing the facility. VD thanks the European Regional Development Fund - Project ENOCH (CZ.02.1.01/0.0/0.0/16_019/0000868) for

\section{References}

[1] P.B. Sampson, Y. Liu, B. Forrest, G. Cumming, S.-W. Li, N.K. Patel, L. Edwards, R. Laufer, M. Feher, F. Ban, D.E. Awrey, G. Mao, O. Plotnikova, R. Hodgson, I. Beletskaya, J.M. Mason, X. Luo, V. Nadeem, X. Wei, R. Kiarash, B. Madeira, P. Huang, T.W. Mak, G. Pan, H.W. Pauls, The Discovery of Polo-Like Kinase 4 Inhibitors: Identification of (1R,2S)-2-(3-((E)-4-(((cis)-2,6-

Dimethylmorpholino)methyl)styryl)-1H-indazol-6yl)-5'-methoxyspiro[cyclopropane-1,3'-indolin]-2'one (CFI-400945) as a Potent, Orally Active Antitumor Agent, J. Med. Chem. 58 (2015) 147169. https://doi.org/10.1021/jm5005336

[2] C. Cui, H. Kakeya, H. Osada, A novel mammalian cell cycle inhibitor produced by Aspergillus Fumigatus, J Antibiot Tokyo. 49 (1996) 832-5. https://doi.org/10.7164/antibiotics.49.832

[3] R. Zhou, Q. Wu, M. Guo, W. Huang, X. He, L. Yang, F. Peng, G. He, B. Han, Organocatalytic cascade reaction for the asymmetric synthesis of novel chroman-fused spirooxindoles that potently inhibit cancer cell proliferation, Chem. Commun. day standard 3-(4,5-dimethylthiazol-2yl)-5-(3carboxymethoxyphenyl)-2-(4-sulfophenyl)2H-tetrazolium reduction assay in 384-well plates on a robotic high-throughput screening platform (HighResBio, Boston, MA) as described elsewhere [36]. The $\mathrm{IC}_{50}$ values were calculated from the respective dose-response curves of compounds with Dotmatics (San Diego, CA, USA)

support. GM and RG thank Aragen Lifesciences Pvt Ltd. Hyderabad, India, for providing computational resources for synthesising and characterising all the compounds by NMR, LC-MS, and FT-IR.

\section{Supplementary data}

NMR, LC-MS and FT-IR spectra of all compounds are provided as Supplementary Data.

51

(2015)

13113-13116.

https://doi.org/10.1039/C5CC04968G

[4] A. Kamal, R. Mahesh, V.L. Nayak, K.S. Babu, G.B. Kumar, A.B. Shaik, J.S. Kapure, A. Alarifi, Discovery of pyrrolospirooxindole derivatives as novel cyclin dependent kinase 4 (CDK4) inhibitors by catalyst-free, green approach, Eur. J. Med. Chem. $\quad 108 \quad$ (2016) 476-485. https://doi.org/10.1016/j.ejmech.2015.11.046

[5] R. Tokala, S. Thatikonda, S. Sana, P. Regur, C. Godugu, N. Shankaraiah, Synthesis and in vitro cytotoxicity evaluation of $\beta$-carboline-linked 2,4thiazolidinedione hybrids: potential DNA intercalation and apoptosis-inducing studies, New J. Chem. $\quad 42 \quad$ (2018) 16226-16236. https://doi.org/10.1039/C8NJ03248C

[6] M.S. Phull, S.S. Jadav, R. Gundla, P.S. Mainkar, A perspective on medicinal chemistry approaches towards adenomatous polyposis coli and Wnt signal based colorectal cancer inhibitors, Eur. J. Med. Chem. $\quad 212 \quad$ (2021) 113149. https://doi.org/10.1016/j.ejmech.2020.113149 
[7] G. Balan, M.J. Bartlett, J. Chandrasekhar, J.A. Codelli, J.H. Conway, J.L. Cosman, R.V. Kalla, M. Kim, S.H. Lee, J.R. Lo, J.A. Loyer-Drew, S.A. Mitchell, T.D. Perry, G.B. Phillips, P.J. Salvo, J.J. Van Veldhuizen, S.C. Yeung, J. Zablocki, Substituted 6-Azabenzimidazole Compounds Having Hpk1 Inhibitory Activity, 2020. https://patentscope.wipo.int/search/en/detail.jsf?do $\underline{\mathrm{cId}=W O 2020092528}$ (accessed May 13, 2021).

[8] K.R. Senwar, P. Sharma, T.S. Reddy, M.K. Jeengar, V.L. Nayak, V.G.M. Naidu, A. Kamal, N. Shankaraiah, Spirooxindole-derived morpholinefused-1,2,3-triazoles: Design, synthesis, cytotoxicity and apoptosis inducing studies, Eur. J. Med. Chem. 102 (2015) 413-424. https://doi.org/10.1016/j.ejmech.2015.08.017

[9] Y. Zhao, S. Yu, W. Sun, L. Liu, J. Lu, D. McEachern, S. Shargary, D. Bernard, X. Li, T. Zhao, P. Zou, D. Sun, S. Wang, A Potent SmallMolecule Inhibitor of the MDM2-p53 Interaction (MI-888) Achieved Complete and Durable Tumor Regression in Mice, J. Med. Chem. 56 (2013) 55535561. https://doi.org/10.1021/jm4005708

[10] K.S. Lechner, M.F. Neurath, B. Weigmann, Role of the IL-2 inducible tyrosine kinase ITK and its inhibitors in disease pathogenesis, J. Mol. Med. 98 (2020) 1385-1395. https://doi.org/10.1007/s00109-020-01958-Z

[11] X. Liu, C.T. Berry, G. Ruthel, J.J. Madara, K. MacGillivray, C.M. Gray, L.A. Madge, K.A. McCorkell, D.P. Beiting, U. Hershberg, M.J. May, B.D. Freedman, T Cell Receptor-induced Nuclear Factor $\kappa \mathrm{B}(\mathrm{NF}-\kappa \mathrm{B})$ Signaling and Transcriptional Activation Are Regulated by STIM1- and Orai1mediated Calcium Entry, J. Biol. Chem. 291 (2016) 8440-8452.

https://doi.org/10.1074/jbc.M115.713008

[12] L. Youssefian, H. Vahidnezhad, M. Yousefi, A.H. Saeidian, A. Azizpour, A. Touati, N. Nikbakht, K.K.- Hesari, M.M. Adib-Sereshki, S. Zeinali, B. Mansoori, A. Jazayeri, R. Karamzadeh, P. Fortina, E. Jouanguy, J.-L. Casanova, J. Uitto, Inherited Interleukin 2-Inducible T-Cell (ITK) Kinase Deficiency in Siblings With Epidermodysplasia Verruciformis and Hodgkin Lymphoma, Clin. Infect. Dis. Off. Publ. Infect. Dis. Soc. Am. $68 \quad$ (2019) 1938-1941. https://doi.org/10.1093/cid/ciy942
[13] A.K. Kannan, N. Sahu, S. Mohanan, S. Mohinta, A. August, IL-2-inducible T-cell kinase modulates TH2-mediated allergic airway inflammation by suppressing IFN- $\gamma$ in naive CD4+ T cells, J. Allergy Clin. Immunol. 132 (2013) 81120.e205. https://doi.org/10.1016/j.jaci.2013.04.033

[14] H.-S. Cho, A. Reboldi, J.A. Hall, L.J. Berg, The Tec kinase ITK is essential for ILC2 survival and epithelial integrity in the intestine, Nat. Commun. 10 (2019) 784-784. https://doi.org/10.1038/s41467-019-08699-9

[15] A.K. Kannan, D.-G. Kim, A. August, M.S. Bynoe, Itk signals promote neuroinflammation by regulating CD4+ T-cell activation and trafficking, J. Neurosci. $\quad 35 \quad$ (2015) 221-233. https://doi.org/10.1523/JNEUROSCI.195714.2015

[16] K. Brameld, T. Owens, Benzimidazole Derivatives as Itk Inhibitors, 2014. https://patentscope.wipo.int/search/en/detail.jsf?do $\underline{\mathrm{cId}=W O 2014036016}$ (accessed February 23, 2021).

[17] J.-D. Charrier, A. Miller, D.P. Kay, G. Brenchley, H.C. Twin, P.N. Collier, S. Ramaya, S.B. Keily, S.J. Durrant, R.M.A. Knegtel, A.J. Tanner, K. Brown, A.P. Curnock, J.-M. Jimenez, Discovery and Structure-Activity Relationship of 3-Aminopyrid-2-ones as Potent and Selective Interleukin-2 Inducible T-Cell Kinase (Itk) Inhibitors, J. Med. Chem. 54 (2011) 2341-2350. https://doi.org/10.1021/jm101499u

[18] J. Das, P. Chen, D. Norris, R. Padmanabha, J. Lin, R.V. Moquin, Z. Shen, L.S. Cook, A.M. Doweyko, S. Pitt, S. Pang, D.R. Shen, Q. Fang, H.F. de Fex, K.W. McIntyre, D.J. Shuster, K.M. Gillooly, K. Behnia, G.L. Schieven, J. Wityak, J.C. Barrish, 2-Aminothiazole as a Novel Kinase Inhibitor Template. Structure-Activity Relationship Studies toward the Discovery of N-(2Chloro-6-methylphenyl)-2-[[6-[4-(2-

hydroxyethyl)-1- piperazinyl)]-2-methyl-4pyrimidinyl]amino)]-1,3-thiazole-5-carboxamide (Dasatinib, BMS-354825) as a Potent pan-Src Kinase Inhibitor, J. Med. Chem. 49 (2006) 68196832. https://doi.org/10.1021/jm060727j

[19] W. Guo, R. Liu, Y. Ono, A.-H. Ma, A. Martinez, E. Sanchez, Y. Wang, W. Huang, A. Mazloom, J. Li, J. Ning, E. Maverakis, K.S. Lam, H.-J. Kung, Molecular characteristics of CTA056, a 
novel interleukin-2-inducible T-cell kinase inhibitor that selectively targets malignant $\mathrm{T}$ cells and modulates oncomirs, Mol. Pharmacol. 82 (2012) 938-947. https://doi.org/10.1124/mol.112.079889

[20] H. Johansson, K. Lawitz, G. Nikitidis, P. Sjö, P. Storm, Use of and Some Novel Imidazopyridines, 2004.

https://patentscope.wipo.int/search/en/detail.jsf?do cId=WO2004016611 (accessed February 23, 2021).

[21] J. Jurcak, M. Barrague, T. Gillespy, M. Edwards, K. Musick, P. Weintraub, Y. Du, R. Dharanipragada, A. Parkar, Thienopyrazoles, US20070254937A1, 2007. https://patents.google.com/patent/US20070254937/ en (accessed February 23, 2021).

[22] D. Riether, R. Zindell, J.A. Kowalski, B.N. Cook, J. Bentzien, S.D. Lombaert, D. Thomson, S.Z. Kugler, D. Skow, L.S. Martin, E.L. Raymond, H.H. Khine, K. O'Shea, J.R. Woska, D. Jeanfavre, R. Sellati, K.L.M. Ralph, J. Ahlberg, G. Labissiere, M.A. Kashem, S.S. Pullen, H. Takahashi, 5Aminomethylbenzimidazoles as potent ITK antagonists, Bioorg. Med. Chem. Lett. 19 (2009) $1588-1591$.

https://doi.org/10.1016/j.bmcl.2009.02.012.

[23] G.A. Flynn, S.A. Lee, M. Faris, D.W. Brandt, S. Chakravarty, ITK inhibitors for treating blood cell malignancies, EP2865381A1, 2015. https://patents.google.com/patent/EP2865381A1/e n (accessed February 23, 2021).

[24] C.M. Alder, I.R. Baldwin, N.P. Barton, A.J. Campbell, A.C. Champigny, J.D. Harling, A.C. Maxwell, J.K. Simpson, I.E.D. Smith, C.J. Tame, C. Wilson, J.M. Woolven, Derivatives of 2-[2-(benzoor Pyrido-) Thiazolylamino]-6-Aminopyridine, Useful in the Treatment of Respiratoric, Allergic or Inflammatory Diseases, 2011. https://patentscope.wipo.int/search/en/detail.jsf?do $\underline{\mathrm{cId}=\mathrm{WO} 2011110575}$ (accessed February 23, 2021).

[25] A.D. Velankar, G. Quintini, A. Prabhu, A. Weber, G. Hunaeus, B. Voland, M. Wuest, C. Orjeda, D. Harel, S. Varghese, V. Gore, M. Patil, D. Gayke, M. Herdemann, I. Heit, A. Zaliani, Synthesis and biological evaluation of novel (4 or 5aryl)pyrazolyl-indoles as inhibitors of interleukin-2 inducible T-cell kinase (ITK), Bioorg. Med. Chem. 18 (2010) 4547-4559. https://doi.org/10.1016/j.bmc.2010.04.056
[26] Y. Zhong, A.J. Johnson, J.C. Byrd, J.A. Dubovsky, Targeting Interleukin-2-Inducible T-cell Kinase (ITK) in T-Cell Related Diseases, Postdoc J. J. Postdr. Res. Postdr. Aff. 2 (2014) 1-11. https://doi.org/10.14304/surya.jpr.v2n6.1

[27] H.-S. Cho, H.M. Shin, H. Haberstock-Debic, Y. Xing, T.D. Owens, J.O. Funk, R.J. Hill, J.M. Bradshaw, L.J. Berg, A Small Molecule Inhibitor of ITK and RLK Impairs Th1 Differentiation and Prevents Colitis Disease Progression, J. Immunol. Baltim. Md 1950. 195 (2015) 4822-4831. https://doi.org/10.4049/jimmunol.1501828

[28] J.M. Fuhriman, M.C.G. Winge, H. HaberstockDebic, J.O. Funk, J.M. Bradshaw, M.P. Marinkovich, ITK and RLK Inhibitor PRN694 Improves Skin Disease in Two Mouse Models of Psoriasis, J. Invest. Dermatol. 138 (2018) 864-871. https://doi.org/10.1016/j.jid.2017.10.029

[29] J.A. Dubovsky, K.A. Beckwith, G. Natarajan, J.A. Woyach, S. Jaglowski, Y. Zhong, J.D. Hessler, T.-M. Liu, B.Y. Chang, K.M. Larkin, M.R. Stefanovski, D.L. Chappell, F.W. Frissora, L.L. Smith, K.A. Smucker, J.M. Flynn, J.A. Jones, L.A. Andritsos, K. Maddocks, A.M. Lehman, R. Furman, J. Sharman, A. Mishra, M.A. Caligiuri, A.R. Satoskar, J.J. Buggy, N. Muthusamy, A.J. Johnson, J.C. Byrd, Ibrutinib is an irreversible molecular inhibitor of ITK driving a Th1-selective pressure in T lymphocytes, Blood. 122 (2013) 2539-2549. https://doi.org/10.1182/blood-2013-06-507947

[30] G. Mudasani, K. Paidikondala, R. Gundla, S. Joseph Maddirala, V. Das, Synthesis and Biological Evaluation of 5'-Arylspiro[piperidine-4,3'-pyrrolo[2,3-b]pyridin] Analogues, ChemistrySelect. 6 (2021) 3378-3381. https://doi.org/10.1002/slct.202004719

[31] L. He, D. Li, C. Zhang, P. Bai, L. Chen, Discovery of (R)-5-(benzo[d][1,3]dioxol-5-yl)-7((1-(vinylsulfonyl)pyrrolidin-2-yl)methyl)-7Hpyrrolo[2,3-d]pyrimidin-4-amine (B6) as a potent Bmx inhibitor for the treatment of NSCLC, Bioorg. Med. Chem. Lett. 27 (2017) 4171-4175. https://doi.org/10.1016/j.bmcl.2017.07.009

[32] Y.-O. Teng, H.-Y. Zhao, J. Wang, H. Liu, M.L. Gao, Y. Zhou, K.-L. Han, Z.-C. Fan, Y.-M. Zhang, H. Sun, P. Yu, Synthesis and anticancer activity evaluation of 5-(2-carboxyethenyl)-isatin 
derivatives, Eur. J. Med. Chem. 112 (2016) 145156. https://doi.org/10.1016/j.ejmech.2015.12.050

[33] S. Mamand, R.L. Allchin, M.J. Ahearne, S.D. Wagner, Comparison of interleukin-2-inducible kinase (ITK) inhibitors and potential for combination therapies for T-cell lymphoma, Sci. Rep. 8 (2018) 14216-14216. https://doi.org/10.1038/s41598-018-32634-5

[34] A. Turetsky, E. Kim, R.H. Kohler, M.A. Miller, R. Weissleder, Single cell imaging of Bruton's Tyrosine Kinase using an irreversible inhibitor, Sci. Rep. $4 \quad$ (2014) 4782. https://doi.org/10.1038/srep04782

[35] O. Parting, S. Langer, M.K. Kuepper, C. Wessling, S. Li, T. Braunschweig, N. Chatain, T. Maié, I.G. Costa, M. Crysandt, M. Huber, T.H. Brümmendorf, S. Koschmieder, M. Schemionek, Therapeutic inhibition of Fc $\gamma \mathrm{RIIb}$ signaling targets leukemic stem cells in chronic myeloid leukemia, Leukemia. $\quad 34 \quad$ (2020) 2635-2647. https://doi.org/10.1038/s41375-020-0977-8

[36] B. Grüner, J. Brynda, V. Das, V. Šícha, J. Štěpánková, J. Nekvinda, J. Holub, K. Pospíšilová, M. Fábry, P. Pachl, V. Král, M. Kugler, V. Mašek, M. Medvedíková, S. Matějková, A. Nová, B. Lišková, S. Gurská, P. Džubák, M. Hajdúch, P. Řezáčová, Metallacarborane Sulfamides: Unconventional, Specific, and Highly Selective Inhibitors of Carbonic Anhydrase IX, J. Med. Chem. $62 \quad$ (2019) 9560-9575. https://doi.org/10.1021/acs.jmedchem.9b00945 